\title{
Recognizing Patterns of Dynamic Behaviors Based on Multiple Relations in Soccer Robotics Domain
}

\author{
Huberto Ayanegui and Fernando Ramos \\ ITESM Campus Cuernavaca \\ Reforma 182-A, Col Lomas De Cuernavaca, \\ 62589, Temixco Morelos, Mexico \\ \{huberto.ayanegui, fernando.ramos\}@itesm.mx
}

\begin{abstract}
This work is focused on the recognition of team patterns represented by different formations played by a soccer team during a match. In the soccer domain, the recognition of formation patterns is difficult due to the dynamic and real time conditions of the environment as well as the multiple interactions among team mates. In this work, some of these multiple interactions are modeled as relations represented by a topological graph which is able to manage the dynamic changes of structures. Thus, the topological graph serves to recognize apparent changes of formations from real changes of them. The proposed model has been tested with different teams in different matches of the Robocup Simulation League. The results have shown that the model can recognize the different main formations used by a team during a match even the multiple changes of the players due to the dynamic nature of a match.
\end{abstract}

Keywords: Pattern recognition, robotic soccer, formations, dynamic behavior.

\section{Introduction}

Formations are the way a soccer team lines up its defense, midfield, and attack line during a match. When talking about formations, defenders are listed first and then midfielders and forwards. For example, a code 4:4:2 represents a formation composed by four defenders, four midfielders, and two forwards. As in the real soccer game the goalkeeper is not considered as part of the formation. Certainly, the dynamic conditions of the soccer game difficult the task of building adequate representations able to facilitate the recognition of formation patterns.

Usually, teams playing in strategic and organized ways search for respecting predefined patterns or formations 2] 3. The purpose of this work is the recognition of patterns represented by formations played by a team during a match. If the recognition task is performed on an opponent team, a soccer team can obtain advantages over it [8]. Visser and colleagues [3] recognize formations of opponent teams using neural networks. This work feeds the observed player positions into a neural network and tries to classify them into a predefined set of formations.

A. Ghosh, R.K. De, and S.K. Pal (Eds.): PReMI 2007, LNCS 4815, pp. 33-40, 2007.

(C) Springer-Verlag Berlin Heidelberg 2007 
If a classification can be done, the appropriate counter formation is looked up and communicated to the players. Due to the fact that Visser does not represent the multiple relations between players, so the neural network has a low level of accuracy for some cases of soccer teams.

Riley and colleagues in 4] use a model to identify 'home areas' of players to recognize formations. A home area specifies the region of the field in which the agent should generally be. Thus, they propose that identifying home areas, the agents can infer a role in the team (defender, midfielder or forward players). A drawback of this approach is that due to dynamic conditions of the world, the player movements can generate such a wide range extending considerably the home areas, which difficult the task of determining the role of a player, therefore a correct formation.

Kuhlmann and colleagues [1] learn team formations similar to Riley and Veloso [4, using home positions. They model the formation as a home position $(\mathrm{X}, \mathrm{Y})$ and introduce a ball attraction vector $(\mathrm{BX}, \mathrm{BY})$ for each player. The $\mathrm{X}$ and $\mathrm{Y}$ values are calculated as the average $\mathrm{x}$ and $\mathrm{y}$ coordinates of the observed player during the course of the game. Values for BX and BY were handpicked for each position and were found through brief experimentation. A weakness of this work is that the home positions have to be adjusted, for some cases, manually.

It is presented in this work an efficient model to recognize patterns of formations based on a representation that takes into account multiple relations among defender, midfielder and forward players. The test domain for this research is simulated robotic soccer, specifically, the Soccer Server System [6], used in the Robot World Cup Initiative [7, an international AI and robotics research initiative. The system is a rich multiagent environment including fully distributed team agents in two different teams composed of eleven agents.

\section{The Multiple Relation Model}

The focus of this work is on teams that play following patterns of high level of abstraction based on a distribution of zones named Defensive (D), Middle (M) and Attack (A), as in classic soccer game. These patterns will be represented as follows: D:M:A. Due to the dynamic conditions of the soccer game, the players are in constant movement and temporally breaking the alignment of players belonging to a zone. To handle the constant changes without an expressive representation of the relations between players can result in an inefficient way of recognizing formations submitted to a dynamic environment. In the next sections will be explained how the zones and the players belonging to them are recognized.

\subsection{Recognition of Team Zones}

As in human soccer domains the players in robotic soccer should tend to be organized [1, 9]. That is, each player has a strategic position that defines its movement range in the soccer field. The role of a player is quite related with 
a predefined area within which an individual player can play basically in the field. Any behaviours of a player depend on its current role. According to the position of the player, roles in robotic soccer can be divided into four types: goalkeeper, defenders, midfielders and forwards. Different roles are associated with different positions and different behaviours that players assume. However, due to the dynamic changing conditions of a match, a defender could become a forward temporarily as his team is trying to attack. As well, a forward could become a defender temporarily when his team is being attacked. So the roles of a player are dynamically changing and a player can have dynamic behaviours in a match. Consequently, the recognition of formation patterns is difficult due to the dynamic and real time conditions of the environment. It is needed to determine, first of all, the belonging of players to a specific zone. This first approach tells us the clustering of players to a zone. In this work, the clustering algorithm, K-means [10], is applied to meet this first stage. K-means classifies a given data set through a certain number of clusters (assume $\mathrm{k}$ clusters) fixed a priori. In this work, $\mathrm{k}=3$ such that three zones will be defined: defensive, middle and attack zones (D:M:A). From the log file (game film), the data from one team is extracted and K-means is applied in each simulation cycle of the game. The positions of each player, with respect to the $\mathrm{x}$ axis, are taken as the input of the clustering algorithm and the output of clustering is the classification, according to their $\mathrm{x}$ position, of all players of the team in the three clusters. Due to the continuously moving of players, it is not feasible to conclude what players are in each zone of a team from a single cycle only, a period of time should be considered.

Clustering algorithm is useful to determine the three zones of a team but it is not able to represent the multiple relations between players of each zone. Given that patterns of formations are based on relations that determine structures then an additional model is crucial for the recognition of patterns of formations. Such model has to be sufficiently robust in order to manage the constant positional changes of players. The next section describes how a set of triangular sub-graphs connected together, that uses as input the result of K-means algorithm, can be used as an adequate representational model able to facilitate the recognition of formation patterns.

\subsection{Representation of Multiple Relations}

A formation is represented by a set of relations between players. Thus, the relations represent the structure that supports a formation. So, a change of relations between players entails a change of formation. It is needed at least the change of one relation to transform one structure into another one. Constant changes of relations could occur because the multiple relations in a formation and the dynamic nature of a match. Figure 1(a) illustrates the relations of each one of the players with the rest of their teammates. A total of 90 relations are obtained by given by the formula: $n(n-1)$, where $n$ represents the number of players. This formula considers two relations by each pair of players. Thus, one relation is represented by the link from player A to player B and the second one from player B to player A. For practical reasons it is considered just one of these 


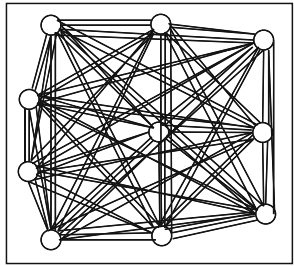

(a)

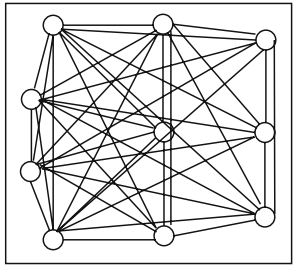

(b)

Fig. 1. All posible relations between players of a soccer team. (a) 90 relations and (b) 45 relations.

relations. Thus, the total of relations is $\frac{n(n-1)}{2}=45$. Figure 1(b) illustrates these 45 relations.

On the one hand, the control of such number of relations becomes very difficult to be managed because any change of relations would produce a change of structure. In addition, it could happen that several changes of relations occur at the same time then the problem of detecting what relations are provoking changes of structures becomes much more difficult to be managed. On the other hand, the 45 relations are not relevant in a real match, because a relevant relation is the one in which a player uses to exchange passes and positions in a strategic way. Thus, a player stays related with his closer neighbor belonging to his zone and the closer neighbor belonging to the neighbor zone. In this work, the goal is to build a simple but robust structure based on relevant relations able to manage the dynamic nature of the game in a topological way, based on triangular sub-graphs that are built as indicated below:

- Step 1. Let $\mathrm{A}=\left\{a_{i}<b_{i}<c_{i}<\ldots<n_{i}\right\}$ be finite number of nodes (players) belonging to a zone $Z_{i}$. Where player $a_{i}$ is located at the top of the zone and player $n_{i}$ at the bottom of it. The rest of the nodes are located at intermediate positions. Thus, $a_{i}$ is linked with $b_{i}, b_{i}$ is linked with $c_{i}$, and so on (see Fig. 2(a)).

- Step 2. Once the nodes of the zones have been linked, nodes of neighbor zones based on minimal distances are linked until a planar graph is built (see Fig. 2(b)).

Figure2(c) shows the planar graph represented by triangular sub-graphs as result of applying the previous two steps.

The total number of relations of a graph, which has been built based on the method described above, is given by $N_{m}+15$; where $N_{m}$ is the number of nodes of the middle zone (Due to the lack of space the deduction of this formula is not described in this work). For instance, for a formation 4:4:2, the number of relations will be 19 , because $N_{m}=4$. The advantages of this method are expressed below:

- The number of relations has been reduced from 45 to 19 for the formation 4:4:2. Then, 26 relations have been eliminated. 


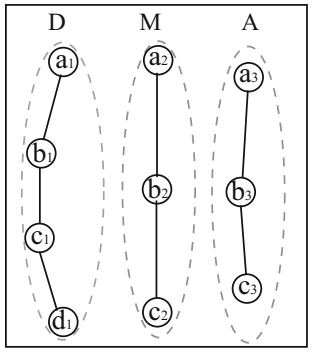

(a)

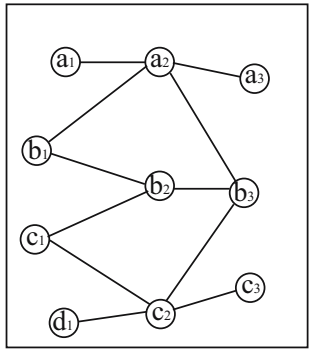

(b)

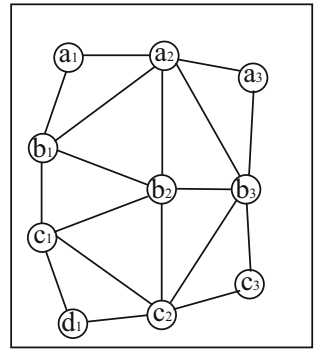

(c)

Fig. 2. (a) Step 1. Neighbor nodes of the same zone are linked. (b) Step 2. Neighbor nodes of neighbor zones are linked. (c) Planar graph obtained from step 1 and step 2.

- In a formation the minimal number of relations of a node is 2 meanwhile the maximal number is 9 . An example of this kind of formation is 9:1:1. For this formation the total number of relations is 16 .

- Triangular subgraphs are able to assume a topological behavior. That is, even if a structure is deformed because positional changes of nodes, the topological property of the triangular graphs helps to preserve the structure.

\section{Pattern Recognition Process}

Fig. 3 shows the process to recognize patterns of formations and changes of structures that support the formations. The first module serves to determine the zones by using a clustering algorithm; the second module builds the multiple relations which are expressed by a topological graph and finally in the third module the changes of structures are detected if topological properties of a defined structure have been broken.

Module 1. Recognition of team zones. The algorithm of clustering is performed during the first cycles of the match and it is stopped until the number of players in each group does not change. In this way, the three zones of a team, defensive, middle and attack zones are recognized.

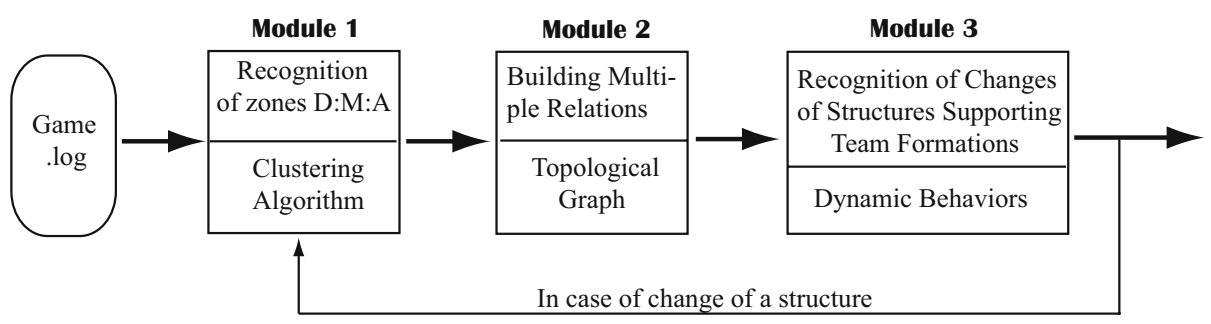

Fig. 3. Process to recognize pattern formations 
Module 2. Building multiple relations and a topological graph. Based on the three zones recognized by the clustering algorithm, relevant multiple relations and a topological planar graph are built.

Module 3. Recognition of Changes of Structures that support Team Formations. Changes of structures are detected if topological properties of a defined structure have been broken. A topological graph is by definition a planar graph. In a planar graph any pair of nodes can be linked. In addition, any link of the graph should not be intersected by any other link. Otherwise, if the topological property of the graph has been broken then another structure supporting a formation should be built. An example of intersected links is shown in Figure 4(b). Intersections occur when players change their roles in order to build a new formation or due to reactive behavior in response to the opponent. If intersections of links occur during a short period of time, thus they have not considered as a change of formation. But, if they occur during a long period of time, clustering algorithm should redefine the zones and a new topological graph should be built. Fig. 4(b).

\section{Experimental Results}

In this section, important experimental results are shown. The results to be shown are derived from a match of the FC Portugal soccer team of the RoboCup Simulation Championship. This team has won several world RoboCup championships. However, in order to valid the approach presented in this work a vast number of matches has been analyzed.

Fig. 4 shows the results of the clustering algorithm derived from the first 1500 cycles of a match. As you can see, the clustering algorithm recognized the three zones of a team. 10 different classifications (formations) have been detected. However, formation 4.3:3 has been recognized more times than others. In particular, it has been recognized most frequently during the first 1000 cycles. The formation 4:2:2 was recognized in the next 500 cycles. On the contrary,

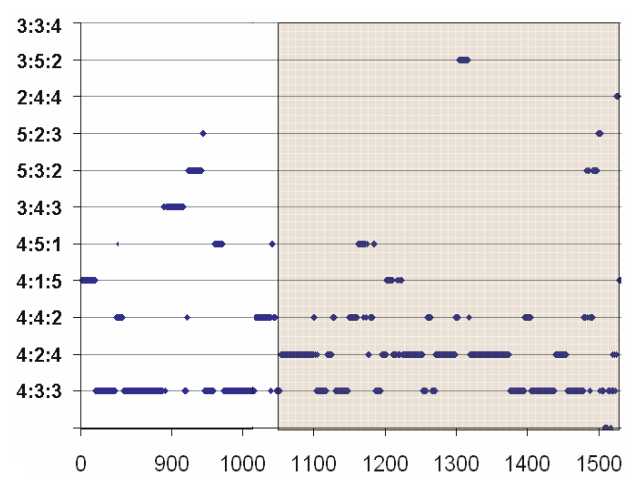

Fig. 4. Clustering results of the first 1500 simulation cycles 

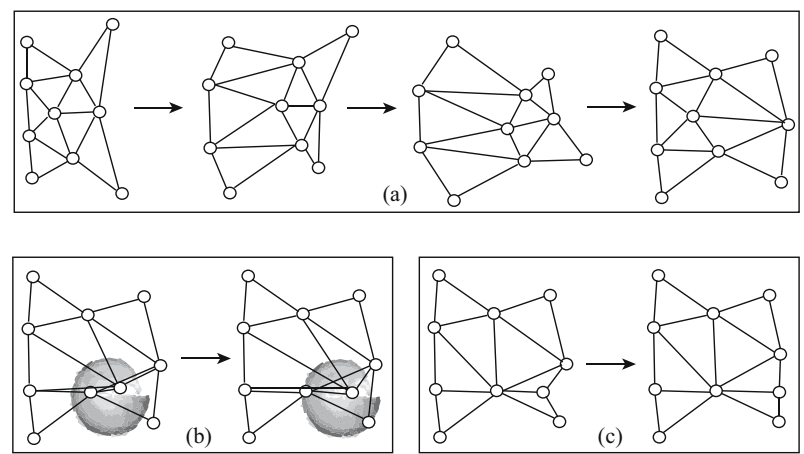

Fig. 5. (a) Four frames showing a plannar graph during the first 1000 simulation cycles. (b) Intersection of relations (shadow region). (c) Building of a new plannar graph for a new formation.

formations such as 3:5:2, 2:4:4, 5:2:3, and 5:3:2 have been detected during a very short period of time.

Fig. 5(a) shows the 9 triangles of the planar graph of some simulation cycles during the first 1000 simulation cycles. It is observed from this sequence of graphs that the structured supporting the formation 4:3:3 is maintained. However, the clustering algorithm recognized different formations even that the relations between agents have not changed. The team started with the formation 4:3:3 and changed to 4:2:4 at the cycle 1050, just after scoring the second goal. A possible cause could be that the score was in favour of this team, so the team has possibly decided to experiment a more offensive behaviour. Fig. 5(b) shows intersections of relations that indicates a change of formation. Once intersections of links have been detected the relations are redefined and a new formation is determined as describe by module 2 discussed before. Fig. 5(c) shows the new planar graph built because the intersections shown in Fig. [5(b).

\section{Conclusion}

In this work, we have been concerned with the recognition of pattern dynamic behaviors within complex, competitive and real time domains, such as soccer robotic games. The patterns to be recognized are formations that can change because strategic reasons or due to reactive behavior in response to the opponent. These facts bring about multiple interactions between agents of a team, and make difficult the task of recognizing formation patterns. In this work, we presented an efficient model to recognize formations based on a rich representation that takes into account multiple relations among players, including the neighbourhood relation between players of the same zone and the neighbourhood relation between players belonging to neighbour zones. In particular, a topological model, based on triangular sub-graphs, has been built, which is able to manage the deformations of structures due to the dynamic changes and the 
multiple relations. A vast number of matches have been analyzed to test the model. The experiments have shown that the model is able to recognize formations and apparent and real changes of them.

As future work variants around a formation will be modelled. A variant is defined as a temporal change around a given formation during a short period of time. We consider that variants are, in most of the times, the strategic key of the teams that decide the final result of a match.

\section{References}

1. Kaminka, G., A., Fidanboylu, M., Allen, C., Veloso, M.: Learning the Sequential Coordinated Behavior of Teams from Observations. In: Proceedings of the RoboCup-2002 Symposium, Fukuoka, Japan (June 2002)

2. Kuhlmann, G., Stone, P., Lallinger, J.: The Champion UT Austin Villa 2003 Simulator Online Coach Team. In: Polani, D., Browning, B., Bonarini, A., Yoshida, K. (eds.) RoboCup 2003. LNCS (LNAI), vol. 3020, Springer, Heidelberg (2004)

3. Ubbo, V., Christian, D., Sebastian, H., Esko, S.: Weland Hans-Georg: Recognizing Formations in Opponent Teams. In: Stone, P., Balch, T., Kraetzschmar, G.K. (eds.) RoboCup 2000. LNCS (LNAI), vol. 2019, Springer, Heidelberg (2001)

4. Patrick, R., Manuela, V., Gal, K.: An empirical study of coaching. In: Distribuited Autonomous Robotic Systems 6, Springer, Heidelberg (2002)

5. Crelle, A.L.: Sammlung mathematischer Aufstze und Bemerkungen, vol. 1. Maurer Berlin, p. 176 (1821)

6. Itsuki, N., lan, F.: Investigating the Complex with Virtual soccer. In: Heudin, J.-C. (ed.) VW 1998. LNCS (LNAI), vol. 1434, pp. 241-253. Springer, Heidelberg (1998)

7. Kitano, H., Tambe, M., Stone, P., Veloso, M., Coradeschi, S., Osawa, E., Matsubara, Noda, I., Asada, M.: The RoboCup Synthetic Agent Challenge 1997. In: Proceedings of IJCAI 1997, Nagoya, Japan (August 1997)

8. David, C., Shaul, M.: Incorporating Opponent Models into Adversary Search. In: Thirteenth National Conference on Artificial lntelligence, Portland Oregon, AAAI Press (1996)

9. Bo, Y., Qinghua, W.: Agent brigade in dynamic formation of robotic soccer. In: Proceedings of the 3rd World Congress on Intelligent Control and Automation, vol. 1, pp. 174-178 (2000)

10. MacQueen, J.B.: Some Methods for classification and Analysis of Multivariate Observations. In: Proceedings of 5th Berkeley Symposium on Mathematical Statistics and Probability, Berkeley, vol. 1, pp. 281-29. University of California Press (1967)

11. Kuhlmann, G., Stone, P., Lallinger, J.: The UT Austin Villa 2003 Champion Simulator Coach: A Machine Learning Approach. In: Nardi, D., Riedmiller, M., Sammut, C., Santos-Victor, J. (eds.) RoboCup 2004. LNCS (LNAI), vol. 3276, pp. 636-644. Springer, Heidelberg (2005) 